\title{
COVID-19 associated coagulopathy in critically ill patients: A hypercoagulable state demonstrated by parameters of haemostasis and clot waveform analysis
}

\author{
Bingwen Eugene Fan ${ }^{1,2,9,10}$. Jensen $\mathrm{Ng}^{3,9,10}$. Stephrene Seok Wei Chan ${ }^{1,2,9,10}$. Dheepa Christopher ${ }^{1,2,9,10}$. \\ Allison Ching Yee Tso ${ }^{1,2,9,10}$ • Li Min Ling ${ }^{4,5,9,10}$. Barnaby Edward Young 4,5,9,10 . Lester Jun Long Wong ${ }^{1}$. \\ Christina Lai Lin Sum ${ }^{6} \cdot$ Hwee Tat Tan ${ }^{6} \cdot$ Mui Kia Ang ${ }^{6} \cdot$ Gek Hsiang Lim $^{7} \cdot$ Kiat Hoe Ong ${ }^{1,2,9,10}$. \\ Ponnudurai Kuperan ${ }^{1,2,9,10}$. Yew Woon Chia ${ }^{8,9,10}$
}

Accepted: 15 October 2020 / Published online: 24 October 2020

(c) Springer Science+Business Media, LLC, part of Springer Nature 2020

\begin{abstract}
Patients with COVID-19 are known to be at risk of developing both venous, arterial and microvascular thrombosis, due to an excessive immuno-thrombogenic response to the SARS-CoV-2 infection. Overlapping syndromes of COVID-19 associated coagulopathy with consumptive coagulopathy and microangiopathy can be seen in critically ill patients as well. Blood was collected from 12 Intensive Care Unit (ICU) patients with severe COVID-19 who were on either mechanical ventilation or on high flow oxygen with a $\mathrm{PaO} 2 / \mathrm{FiO} 2$ ratio of $<300 \mathrm{mmHg}$. Laboratory tests were performed for parameters of haemostasis, clot waveform analysis and anti-phospholipid antibodies. CWA parameters were raised with elevated aPTT median Min1 (clot velocity) 9.3\%/s (IQR 7.1-9.9\%/s), elevated PT median Min1 10.3\%/s (IQR 7.1-11.1\%/s), elevated aPTT median Min2 (clot acceleration) $1.5 \% / \mathrm{s}^{2}$ (IQR 1.0-1.6\%/ $\mathrm{s}^{2}$ ), elevated PT median Min2 5.2\%/s $\mathrm{s}^{2}\left(3.6-5.7 \% / \mathrm{s}^{2}\right)$, elevated aPTT median Max2 (clot deceleration) $1.3 \% / \mathrm{s}^{2}$ (IQR $0.8-1.4 \% / \mathrm{s}^{2}$ ) elevated PT median Max2 3.8\%/ $\mathrm{s}^{2}$ (IQR 2.6-4.2\%/ $\mathrm{s}^{2}$ ), increased aPTT median Delta change (decreased light transmission due to increased clot formation) 87.8\% (IQR 70.2-91.8\%) and PT median Delta change $33.0 \%$. This together with raised median Factor VIII levels of $262.5 \%$, hyperfibrinogenemia (median fibrinogen levels $7.5 \mathrm{~g} / \mathrm{L}$ ), increased median von Willebrand factor antigen levels $320 \%$ and elevated median D-dimer levels $1.7 \mu \mathrm{g} / \mathrm{dl}$ support the diagnosis of COVID-19 associated coagulopathy. A lupus anticoagulant was present in 50\% of patients. Our laboratory findings further support the view that severe SARS-CoV-2 infection is associated with a state of hypercoagulability.
\end{abstract}

Keywords Hypercoagulability $\cdot$ Coronavirus $\cdot$ Thrombosis $\cdot$ Sepsis $\cdot$ Thrombophilia

Senior authors Ponnudurai Kuperan and Yew Woon Chia contributed equally.

Bingwen Eugene Fan

Bingwen_Eugene_Fan@ttsh.com.sg

1 Department of Haematology, Tan Tock Seng Hospital, Singapore, Singapore

2 Department of Laboratory Medicine, Khoo Teck Puat Hospital, Singapore, Singapore

3 Department of Anaesthesiology, Intensive Care and Pain Medicine, Tan Tock Seng Hospital, Singapore, Singapore

4 Department of Infectious Diseases, Tan Tock Seng Hospital, Singapore, Singapore
5 National Centre for Infectious Diseases, Singapore, Singapore

6 Department of Laboratory Medicine, Tan Tock Seng Hospital, Singapore, Singapore

7 Clinical Research and Innovation Office, Tan Tock Seng Hospital, Singapore, Singapore

8 Department of Cardiology, Tan Tock Seng Hospital, Singapore, Singapore

9 Lee Kong Chian School of Medicine, Singapore, Singapore

10 Yong Loo Lin School of Medicine, Singapore, Singapore 


\section{Highlights}

- COVID-19 is associated with a hypercoagulable state in critically ill patients.

- Clot waveform analysis shows good correlation with increased levels of factor VIII, fibrinogen, vWFAg and D-dimer, reflecting hypercoagulability in COVID-19 patients.

- Major bleeding can occur in critically ill patients with severe COVID-19.

- Clinical trials including risk prediction models for venous thromboembolism and arterial thrombosis are required, these may incorporate of parameters of haemostasis and global haemostatic tests to establish appropriate thromboprophylactic regimes.

\section{Introduction}

The COVID-19 pandemic has to date claimed more than a million lives, with high mortality contributed in part by thromboembolic disease. Patients with COVID-19 are known to be at risk of developing venous, arterial and microvascular thrombosis, due to an excessive immunothrombogenic response to the SARS-CoV-2 infection [1, 2]. This is secondary to COVID-19 associated coagulopathy, which is a hypercoagulable state characterized by several guidelines [3-5] as having laboratory features of raised D-dimer, Factor VIII and hyperfibrinogenemia, with the potential contributory mechanisms including vasculitis [6], hyperinflammation (raised IL-1-Beta, IL-6 and TNF alpha), neutrophil extracellular traps and hyperviscosity, resulting in both systemic and local macro- and microthrombosis. Antiphospholipid antibodies can also be present transiently, although it is not yet proven if this translates to a clinical hypercoagulable state. Potential overlaps of COVID-19-associated coagulopathy with disseminated intravascular coagulopathy and microangiopathy can also be seen in critically ill patients.

Critically ill patients with COVID-19 have been evaluated with conventional haemostasis tests [7]' as well as global haemostatic tests. Thromboelastography (TEG) in two case series [8,9] and in another case series on rotational thromboelastometry (ROTEM) [10] confirmed a state of hypercoagulopathy with associated thrombosis. Tan et al. [11] described 3 critically ill COVID-19 patients with raised clot waveform parameters suggestive of a hypercoagulable state in COVID-19 infection with no thrombotic events described. We present a series of 12 critically ill COVID-19 patients who were not on thromboprophylaxis or anticoagulation at the time of assessment, where standard haemostatic tests were performed and correlated with clot waveform analysis, a global haemostatic assay [12, 13].

\section{Methods}

In this retrospective observational study, we evaluated 12 Intensive Care Unit (ICU) patients with severe SARS-CoV-2 infection at the National Centre for Infectious Diseases, Singapore, who were on either mechanical ventilation or high flow oxygen with a $\mathrm{PaO} 2 / \mathrm{FiO} 2$ ratio of $<300 \mathrm{mmHg}$. A confirmed case of SARS-CoV-2 infection was defined with a positive result on real time reverse-transcriptase-polymerase-chain-reaction assay of nasopharyngeal swab specimens obtained. The majority of our COVID-19 patients, particularly ambulant and non-oxygen dependent are not routinely administered pharmacological thromboprophylaxis during their hospitalization due to the perceived lower incidence of venous thromboembolism (VTE) in our predominantly Asian population [14].

The 12 patients were assessed between Day 9 to Day 20 of their illness and were started on mechanical thromboprophylaxis as per ICU VTE protocols but were not on any pharmacological thromboprophylaxis at the time of evaluation. Ten patients were referred by the ICU team for advice on initiating pharmacological thromboprophylaxis given their prolonged activated partial thromboplastin time (aPTT) and/or prothrombin time (PT), and 2 were referred at clinical presentation of a suspected lower limb ischaemia. Approximately $10 \mathrm{mls}$ of whole blood was obtained per patient for evaluation. The patients were then followed up for any thromboembolic or bleeding events.

Tests of haemostasis All coagulation tests including lupus anticoagulant were performed on ${ }^{\text {STA }}$ R Max Series coagulation analyzer (Diagnostica Stago, France). PT was measured with ${ }^{\text {STA }}$ Neoplastine CI Plus 10, aPTT with ${ }^{\text {STA Cephas- }}$ creen 10, fibrinogen (modified Clauss) with ${ }^{\text {STA }}$ Liquid FIB, D-dimer with ${ }^{\text {STA }}$ Liatest D-Di and thrombin clotting time with ${ }^{\text {STA }}$ Thrombin 10. Clotting factor levels (Factors II, V, VII, VIII, IX, X, XI) were measured with ${ }^{\text {STA }}$ Deficient II, V, VII, VIII, IX, X, and XI respectively. Von Willebrand factor (vWF) antigen was assayed with immunoturbidimetric method using ${ }^{\text {STA }}$ Liastest $\mathrm{vWF}$ : Ag kit. Both assays for protein $\mathrm{C}$ and anti-thrombin III are functional chromogenic assays using ${ }^{\text {STA }}$ Stachrom protein $\mathrm{C}$ and ${ }^{\text {STA }}$ Stachrom ATIII kits respectively. Protein $\mathrm{S}$ assay is a functional clotting assay using a ${ }^{\text {STA }}$ Staclot protein $\mathrm{S}$ kit. Lupus anticoagulant was performed using STA Staclot DRVV Screen, STA Staclot DRVV Confirm and PTT-LA. Anti-cardiolipin IgM and IgG were quantified using Inova test kits, and 
anti-B2-glycoprotein-1, Euroimmun test kit, both were performed on the Inova Quanta-lyser 3000 analyzer.

Clot waveform analysis CWA was performed with Sysmex CN-6000 automated coagulation analyser (Sysmex Corporation, Kobe, Japan) with Dade Actin FSL (Siemens Healthcare, Marburg, Germany) for aPTT CWA and Innovin (Siemens Healthcare, Marburg, Germany) for PT CWA, as per International Society of Haemostasis and Thrombosis (ISTH) Scientific and Standardization Committee recommendation [15]. Four quantitative parameters were recorded"Min1" (maximum velocity), "Min2" (maximum acceleration), "Max2" (maximum deceleration), and "Delta change" (difference between initial maximum and final maximum values of light transmittance).

\section{Statistical analysis}

Haemostatic evaluation of the patients included tests of haemostasis, CWA, and anti-phospholipid antibodies. We analyzed the aPTT and PT CWA parameters in ICU patients and healthy subjects using mean and standard deviation, with analysis of their differences using the Wilcoxon rank-sum test. Statistical analysis was performed with STATA 16.1. A waiver of informed consent was provided by the Ministry of Health (Singapore) for this study, under the provisions of the Infectious Diseases Act.

\section{Results}

The haemostatic profiles of 12 ICU patients with severe COVID-19 pneumonia were assessed between Day 9 to Day 20 of their illness. Majority were male (11 out of 12), with a median age of 52 years old, with a multiethnic background (Chinese, Indian, Malay and Bangladeshi). The median BMI was raised at 25.7 with patients having comorbidities of hypertension (50\%), diabetes (41.7\%) and hyperlipidemia (33.3\%). The median onset of symptoms to ICU admission was 8.5 days (Interquartile range (IQR) 8-12 days) with a median SOFA score of 4 (IQR 2-6 points) on ICU admission. The median $\mathrm{PaO} 2 / \mathrm{FiO} 2$ ratio on admission to ICU was 168 (IQR 122-203) with 9 patients (75\%) requiring mechanical ventilation. The median haemoglobin concentration was $11.9 \mathrm{~g} / \mathrm{dl}$ (IQR $10.5-13.3 \mathrm{~g} / \mathrm{dl}$ ), with a normal median white blood cell count of $9.4 \times 10^{9} / \mathrm{L}$ (IQR 8.2-13.6 g/dl), lymphopenia of $0.9 \times 10^{9} / \mathrm{L}\left(0.6-1.1 \times 10^{9} / \mathrm{L}\right)$ and a raised median lactate dehydrogenase of $754 \mathrm{U} / \mathrm{L}$ (IQR 714-1098 U/L). The median Padua prediction score for risk of VTE was 5. The median length of ICU stay was 11.5 days (IQR 4.5-24.5 days) and median length of hospital stay 25 days (IQR 21-34.5 days). Table 1 summarizes the demographic and clinical characteristics of these 12 Covid-19 patients in
ICU. Table 2 highlights the multiple quantitative parameters that were recorded.

After risk assessment, all 12 patients were started on pharmacological thromboprophylaxis given their high risk of VTE and evidence for hypercoagulability from haemostatic tests and CWA. For the 2 patients with acute ischaemic limb (Fig. 1a and Case report by Fan et al. [16]), anticoagulation with unfractionated heparin was commenced immediately after blood draw and surgical thrombectomy was successfully performed without bleeding complications. Two older patients suffered from major bleeding- one had recurrent gastrointestinal tract bleeding requiring packed red cell transfusion during the first bleeding episode on day 11 of thromboprophylaxis and later dying from multiorgan failure on Day 38 of illness, and the other succumbing to intracranial haemorrhage on Day 4 of IV unfractionated heparin started for extracorporeal membrane oxygenation (ECMO). During initial assessment of their hemostatic profiles (Fig. 1b, c), these 2 patients were found to have concurrent bacterial infection where 1 had bacterial pneumonia with a raised procalcitonin of $10.4 \mathrm{ug} / \mathrm{L}$, and the second had Stenotrophomonas maltophilia bacteraemia with ventilatorassociated pneumonia. Both had bleeding complications which may have been caused by consumption of clotting factors and platelets caused by micro and macrovascular pulmonary thrombosis (pulmonary intravascular coagulopathy [17]) reflected by the markedly raised D-dimer levels $>20 \mathrm{mg} / \mathrm{dl}$ (these 2 patients were too unwell to undergo CT pulmonary angiography) and possibly exacerbated by consumption from the associated bacterial sepsis. None of the remaining 10 patients had VTE while on thromboprophylaxis during their hospitalization.

Tests of haemostasis PT and aPTT were either slightly prolonged or normal, with median values of $14.6 \mathrm{~s}$ (IQR 14.3-15.8 s) and 38.4 (IQR 34.5-43.6 s) respectively. For additional parameters used to assess DIC by ISTH criteria, median platelet count was normal at $275.5 \times 10^{9}$ (IQR 188 $\left.357 \times 10^{9}\right)$ and median fibrinogen was markedly elevated at $7.5 \mathrm{~g} / \mathrm{L}$ (IQR 5.2-8.5 g/L). The value of D-dimer was markedly increased at $>20 \mu \mathrm{g} / \mathrm{ml}$ for 2 of the patients (16.7\%). Assuming that the D-dimer values for these 2 patients are at $20 \mu \mathrm{g} / \mathrm{ml}$, it suggests that the mean values of the D-dimer was at least 5.7 (7.2) $\mu \mathrm{g} / \mathrm{ml}$ (median, IQR: $2.1,1.4-7.8 \mu \mathrm{g} /$ $\mathrm{ml}$ ) in the study population. None of the patients fulfilled ISTH criteria for DIC.

For quantification of clotting factors, the significant findings were that all 12 patients had elevated Factor VIII levels (median of $262.5 \%$ ), approximately half $(45.5 \%)$ had elevated factor IX levels, and 1 in 3 patients (33.3\%) had an elevated factor II level. All 12 patients also had a markedly elevated vWF antigen level, with median value of $320 \%$ (IQR 259-371\%). Anti-thrombin III levels were 
Table 1 Demographic and Clinical Characteristics of 12 ICU Patients with COVID-19

\begin{tabular}{|c|c|c|c|c|}
\hline & \multicolumn{4}{|c|}{ Total number of patients $(\mathrm{N}=12)$} \\
\hline & No. & $\%$ & Median & IQR \\
\hline Age (years) & & & 52 & 41,61 \\
\hline \multicolumn{5}{|l|}{ Gender } \\
\hline Male & 11 & 91.7 & & \\
\hline Female & 1 & 8.3 & & \\
\hline \multicolumn{5}{|l|}{ Ethnic group } \\
\hline Chinese & 4 & 33.3 & & \\
\hline Malays & 2 & 16.7 & & \\
\hline Indians & 4 & 33.3 & & \\
\hline Others & 2 & 16.7 & & \\
\hline Body mass index; BMI $\left(\mathrm{kg} / \mathrm{m}^{2}\right)$ & & & 25.7 & $23,29.3$ \\
\hline \multicolumn{5}{|l|}{ Comorbidities } \\
\hline Hypertension & 6 & 50.0 & & \\
\hline Diabetes & 5 & 41.7 & & \\
\hline COPD & 1 & 8.3 & & \\
\hline Hyperlipidaemia & 4 & 33.3 & & \\
\hline IHD & 0 & 0.0 & & \\
\hline Renal impairment & 2 & 16.7 & & \\
\hline \multicolumn{5}{|l|}{ Onset of symptoms to hospital admission (days) } \\
\hline Symptoms presented prior admission & & & 3.5 & 3,4 \\
\hline Symptoms presented post admission & & & 11.5 & 0,23 \\
\hline \multicolumn{5}{|l|}{ Onset of symptoms to ICU admission (days) } \\
\hline Symptoms presented prior admission & & & 8.5 & 8,12 \\
\hline Symptoms presented post admission & & & - & - \\
\hline Day of illness at point of haemostatic assessment & & & 13.5 & $11.5,18.5$ \\
\hline SOFA score on ICU admission & & & 4 & 2,6 \\
\hline Padua (VTE) score on ICU admission & & & 5 & 5,6 \\
\hline $\mathrm{PaO}_{2} / \mathrm{FiO}_{2}$ on ICU admission & & & 168 & 122,203 \\
\hline \multicolumn{5}{|l|}{ Mechanical ventilation } \\
\hline No & 3 & 25.0 & & \\
\hline Yes & 9 & 75.0 & & \\
\hline Length of ICU stay (days) & & & 11.5 & $4.5,24.5$ \\
\hline Length of hospital stay (days) & & & 25 & $21,34.5$ \\
\hline \multicolumn{5}{|l|}{ ICU mortality } \\
\hline No & 10 & 83.3 & & \\
\hline Yes & 2 & 16.7 & & \\
\hline \multicolumn{5}{|l|}{ Hospital mortality } \\
\hline No & 10 & 83.3 & & \\
\hline Yes & 2 & 16.7 & & \\
\hline
\end{tabular}

Out of the 2 deaths, 1 was due to bleeding complications mostly normal with a median of $89.5 \%$ (IQR 78-96.5\%). Protein $\mathrm{C}$ and $\mathrm{S}$ were either decreased or at normal levels in most patients (Table 2).

Clot waveform analysis Plasma from the 12 patients were evaluated by CWA on a single occasion. For CWA performed on aPTT, we found a markedly elevated median Min 1 of 9.3\%/s (reference range 2.85-6.65\%/s); elevated median Min2 of $1.5 \% / \mathrm{s}$ (reference range $0.46-1.08 \% / \mathrm{s}$ ) and elevated median Max 2 of 1.3\%/s (reference range $0.37-0.91 \% / \mathrm{s})$. There was a significant decrease in light transmission, represented by a high median delta change of $87.8 \%$ (reference range $10.0-41.1 \%$ ). CWA performed on PT also showed similar marked elevated median Min1 of $10.3 \% / \mathrm{s}$ (reference range 1.96-5.51\%/s); elevated median $\min 2$ of $5.2 \% / \mathrm{s}$ (reference range $0.98-2.84 \% / \mathrm{s}$ ), elevated median Max2 of 3.8\%/s (reference range $0.74-2.28 \% / \mathrm{s}$ ), and elevated median delta change of 33.0 (reference range 
Table 2 Haemostatic, Biochemical and Immunological Profile of the 12 ICU Patients with COVID-19

\begin{tabular}{|c|c|c|c|c|c|c|c|c|}
\hline Biomarkers & Reference Ranges & $\mathrm{n}$ & No. & $\%$ & Median & IQR & Mean & SD \\
\hline Lactate dehydrogenase, LDH (U/L) & $270-550$ & 11 & & & 754 & 714,1098 & 876.5 & 242.8 \\
\hline Thrombin clotting time, TCT (sec) & $15.0-18.0$ & 11 & & & 16.4 & $16.2,17.3$ & 16.8 & 1.2 \\
\hline White blood cell, WBC (x 10*9/L) & $4.0-9.6$ & 12 & & & 9.4 & $8.2,13.6$ & 11.0 & 4.8 \\
\hline Lymphocyte count (x 10*9/L) & $1.1-3.1$ & 12 & & & 0.9 & $0.6,1.1$ & 0.9 & 0.4 \\
\hline Haemoglobin, Hb (g/dL) & & 12 & & & & & & \\
\hline Below reference range & & & 9 & 75.0 & & & & \\
\hline Normal & Males: $13.6-16.6$ & & 3 & 25.0 & & & & \\
\hline Above reference range & Females: $11.8-14.6$ & & 0 & 0.0 & & & & \\
\hline Haemoglobin, Hb (g/dL) & & & & & 11.9 & $10.5,13.3$ & 11.8 & 2.1 \\
\hline Platelets (x 10*9/L) & $150-360$ & 12 & & & & & & \\
\hline Below reference range & & & 3 & 25.0 & & & & \\
\hline Normal & & & 7 & 58.3 & & & & \\
\hline Above reference range & & & 2 & 16.7 & & & & \\
\hline Platelets (x 10*9/L) & & & & & 275.5 & 188,357 & 293.6 & 153.9 \\
\hline Prothrombin time, PT (sec) & $11.7-14.0$ & 12 & & & & & & \\
\hline Below reference range & & & 0 & 0.0 & & & & \\
\hline Normal & & & 1 & 8.3 & & & & \\
\hline Above reference range & & & 11 & 91.7 & & & & \\
\hline Prothrombin time, PT (sec) & & & & & 14.6 & $14.3,15.8$ & 15.0 & 1.4 \\
\hline Activated partial thromboplastin time, aPTT (sec) & $27.0-37.0$ & 12 & & & & & & \\
\hline Below reference range & & & 0 & 0.0 & & & & \\
\hline Normal & & & 5 & 41.7 & & & & \\
\hline Above reference range & & & 7 & 58.3 & & & & \\
\hline Activated partial thromboplastin time (sec) & & & & & 38.4 & $34.5,43.6$ & 40.0 & 6.8 \\
\hline Fibrinogen $(\mathrm{g} / \mathrm{L})$ & $1.8-4.5$ & 12 & & & & & & \\
\hline Below reference range & & & 0 & 0.0 & & & & \\
\hline Normal & & & 2 & 16.7 & & & & \\
\hline Above reference range & & & 10 & 83.3 & & & & \\
\hline Fibrinogen $(\mathrm{g} / \mathrm{L})$ & & & & & 7.5 & $5.2,8.5$ & 6.8 & 1.9 \\
\hline D-dimer $(\mu \mathrm{g} / \mathrm{ml})$ & $<0.50$ & 12 & & & & & & \\
\hline Below reference range & & & 0 & 0.0 & & & & \\
\hline Normal & & & 1 & 8.3 & & & & \\
\hline Above reference range & & & 11 & 91.7 & & & & \\
\hline \multicolumn{9}{|l|}{ D-dimer $(\mu \mathrm{g} / \mathrm{ml})$} \\
\hline$\leq 20$ & & & 10 & 83.3 & 1.7 & $1.4,2.6$ & 2.8 & 2.9 \\
\hline$>20$ & & & 2 & 16.7 & - & - & - & - \\
\hline Factor II (\%) & $70-120$ & 12 & & & & & & \\
\hline Below reference range & & & 3 & 25.0 & & & & \\
\hline Normal & & & 5 & 41.7 & & & & \\
\hline Above reference range & & & 4 & 33.3 & & & & \\
\hline Factor II (\%) & & & & & 107.5 & 74,126 & 100.8 & 31.2 \\
\hline Factor V (\%) & $70-120$ & 11 & & & & & & \\
\hline Below reference range & & & 2 & 18.2 & & & & \\
\hline Normal & & & 7 & 63.6 & & & & \\
\hline Above reference range & & & 2 & 18.2 & & & & \\
\hline Factor V (\%) & & & & & 117 & 93,145 & 123.1 & 44.9 \\
\hline
\end{tabular}


Table 2 (continued)

\begin{tabular}{|c|c|c|c|c|c|c|c|c|}
\hline Biomarkers & Reference Ranges & $\mathrm{n}$ & No. & $\%$ & Median & IQR & Mean & SD \\
\hline Factor VII (\%) & $55-170$ & 10 & & & & & & \\
\hline Below reference range & & & 0 & 0.0 & & & & \\
\hline Normal & & & 9 & 90.0 & & & & \\
\hline Above reference range & & & 1 & 10.0 & & & & \\
\hline Factor VII (\%) & & & & & 98.5 & 92,150 & 119.6 & 39.2 \\
\hline Factor VIII (\%) & $60-150$ & 12 & & & & & & \\
\hline Below reference range & & & 0 & 0.0 & & & & \\
\hline Normal & & & 0 & 0.0 & & & & \\
\hline Above reference range & & & 12 & 100.0 & & & & \\
\hline Factor VIII (\%) & & & & & 262.5 & $196,335.5$ & 284.5 & 102.9 \\
\hline Factor IX (\%): & $60-150$ & 11 & & & & & & \\
\hline Below reference range & & & 0 & 0.0 & & & & \\
\hline Normal & & & 6 & 54.5 & & & & \\
\hline Above reference range & & & 5 & 45.5 & & & & \\
\hline Factor IX (\%) & & & & & 150 & 114,186 & 155.2 & 50.6 \\
\hline Factor X (\%) & $70-120$ & 12 & & & & & & \\
\hline Below reference range & & & 2 & 12.7 & & & & \\
\hline Normal & & & 9 & 75.0 & & & & \\
\hline Above reference range & & & 1 & 8.3 & & & & \\
\hline Factor X (\%) & & & & & 111.5 & $91.5,121$ & 107.6 & 27.9 \\
\hline Factor XI (\%) & $60-150$ & 10 & & & & & & \\
\hline Below reference range & & & 2 & 20.0 & & & & \\
\hline Normal & & & 6 & 60.0 & & & & \\
\hline Above reference range & & & 2 & 20.0 & & & & \\
\hline Factor XI (\%) & & & & & 105.5 & 67,145 & 110.5 & 53.7 \\
\hline von Willebrand factor, vWF (\%) & $56-160$ & 12 & & & & & & \\
\hline Below reference range & & & 0 & 0.0 & & & & \\
\hline Normal & & & 0 & 0.0 & & & & \\
\hline Above reference range & & & 12 & 100.0 & & & & \\
\hline von Willebrand factor, vWF (\%) & & & & & 320 & 259,371 & 308.6 & 71.9 \\
\hline Anti-thrombin III (\%) & $80-130$ & 12 & & & & & & \\
\hline Below reference range & & & 3 & 25.0 & & & & \\
\hline Normal & & & 9 & 75.0 & & & & \\
\hline Above reference range & & & 0 & 0.0 & & & & \\
\hline Antithrombin III (\%) & & & & & 89.5 & $78,96.5$ & 84.4 & 15.1 \\
\hline Protein C (\%) & $70-150$ & 12 & & & & & & \\
\hline Below reference range & & & 5 & 41.7 & & & & \\
\hline Normal & & & 7 & 58.3 & & & & \\
\hline Above reference range & & & 0 & 0.0 & & & & \\
\hline Protein C $(\%)$ & & & & & 78.5 & $46,109.5$ & 77.5 & 37.5 \\
\hline Protein S (\%) & $55-130$ & 12 & & & & & & \\
\hline Below reference range & & & 3 & 25.0 & & & & \\
\hline Normal & & & 8 & 66.7 & & & & \\
\hline Above reference range & & & 1 & 8.3 & & & & \\
\hline Protein S (\%) & & & & & 68 & $47.5,83.5$ & 65.2 & 26.4 \\
\hline Lupus anticoagulant & & 12 & & & & & & \\
\hline Absent & & & 6 & 50.0 & & & & \\
\hline Present & & & 6 & 50.0 & & & & \\
\hline
\end{tabular}


Table 2 (continued)

\begin{tabular}{|c|c|c|c|c|c|c|c|c|}
\hline Biomarkers & Reference Ranges & $\mathrm{n}$ & No. & $\%$ & Median & IQR & Mean & SD \\
\hline Anticardiolipin IgG (GPL units) & $0-20$ & 4 & & & & & & \\
\hline$<20$ & & & 3 & 75.0 & & & & \\
\hline$\geq 20$ & & & 1 & 25.0 & & & & \\
\hline Anticardiolipin IgM (MPL units) & $0-20$ & 4 & & & & & & \\
\hline$<20$ & & & 2 & 50.0 & & & & \\
\hline$\geq 20$ & & & 2 & 50.0 & & & & \\
\hline Anti $\beta 2$ glycoprotein-1 (RU/ml) & $0-20$ & 4 & & & & & & \\
\hline$<2$ & & & 2 & 50.0 & & & & \\
\hline$\geq 2$ & & & 2 & 50.0 & & & & \\
\hline aPTT clot waveform (Min $1, \% / s)$ & $2.86-6.78$ & & & & 9.3 & $7.1,9.9$ & 8.3 & 2.1 \\
\hline aPTT clot waveform (APTT, Min2, \%/s²) & $0.46-1.10$ & & & & 1.5 & $1.0,1.6$ & 1.3 & 0.4 \\
\hline aPTT clot waveform (APTT, Max $2, \% / \mathrm{s}^{2}$ ) & $0.37-0.93$ & & & & 1.3 & $0.8,1,4$ & 1.1 & 0.4 \\
\hline aPTT clot waveform (APTT, delta change \%) & $25.21-63.09$ & & & & 87.8 & $70.2,91.8$ & 82.8 & 11.5 \\
\hline Clot waveform (PT, Min1, \%/s) & $1.95-5.67$ & & & & 10.3 & $7.1,11.1$ & 9.2 & 2.8 \\
\hline Clot waveform (PT, Min2, \%/s²) & $0.97-2.93$ & & & & 5.2 & $3.6,5.7$ & 4.6 & 1.5 \\
\hline Clot waveform (PT, Max $\left.2, \% / \mathrm{s}^{2}\right)$ & $0.75-2.35$ & & & & 3.8 & $2.6,4.2$ & 3.4 & 1.1 \\
\hline Clot waveform (PT, delta change \%) & $6.52-17.28$ & & & & 33.0 & $23.9,37.2$ & 30.7 & 8.9 \\
\hline
\end{tabular}

Reference intervals for clot waveform parameters were established locally based on 124 healthy controls in accordance with the Clinical and Laboratory Standards Institute guidelines

6.6-16.9\%). Comparison of COVID-19 patients with healthy controls showed COVID-19 patients had CWA parameters which were above the upper limit of the respective reference intervals, with statistical significance $(\mathrm{p}<0.001)$ (Tables 3 and 4). There was no biphasic waveform pattern on light transmission curves of the COVID-19 patients that could suggest an underlying DIC [18, 19].

Antiphospholipid antibodies Lupus anticoagulant was present in 6 out of 12 patients $(50 \%)$ which is also prevalent in other series [20,21], with 2 out of 4 patients having mildly raised anticardiolipin antibodies.

\section{Discussion}

Our study further supports the available evidence that severe SARS-CoV-2 infection is associated with hypercoagulability [7, 21]. Clot waveform analysis (CWA) has not been well described as a tool to evaluate a hypercoagulable state, although emerging literature shows promising evidence that this may be useful in predicting for VTE [22] and also in several procoagulable states $[23,24]$. CWA showed a hypercoagulable state with increased parameters of clot maximum velocity ( $\min 1)$, clot maximum acceleration $(\min 2)$, clot maximum deceleration $(\max 2)$ and delta change (decreased light transmission reflective of increased clot thickness) characterizing a hypercoagulable state in 10 patients (with corresponding raised Factor VIII, vWF antigen, D-dimer and fibrinogen levels), with no evidence of concurrent bacterial infection at the time of assessment.

Two patients did not demonstrate a hypercoagulable state on their CWA. These 2 patients had probable pulmonary intravascular coagulopathy (PIC) with bacterial sepsis induced coagulopathy (SIC), and COVID-19-associated coagulopathy with depressed Factor II, X, XI levels and moderate thrombocytopenia. Patients with severe COVID19 who develop SIC would have secondary hyperfibrinolysis with a reduction in fibrinogen levels and platelet counts [25]. Compared with the other 10 patients with a primary hypercoagulable state, they had relatively lower but still elevated levels of fibrinogen, factor VIII and vWF antigen, reflective of the underlying pro-inflammatory state and endothelial activation and dysfunction (resulting in raised vWF antigen levels) from severe COVID-19. Although their CWA showed a mildly increased delta change, the other CWA parameters were either normal or slightly depressed, likely due to the lower levels of clotting factors and fibrinogen due to consumption from both bacterial SIC and PIC when compared to raised CWA parameters (min1, min2, max 2 and increased delta change) in the other 10 patients (see Fig. 1b, c). Collectively, in critically ill COVID-19 patients without a concurrent PIC or SIC, the CWA for both aPTT and PT demonstrate a state of hypercoagulability.

Major bleeding occurred in 2 of our patients, one had recurrent gastrointestinal bleeding while on 


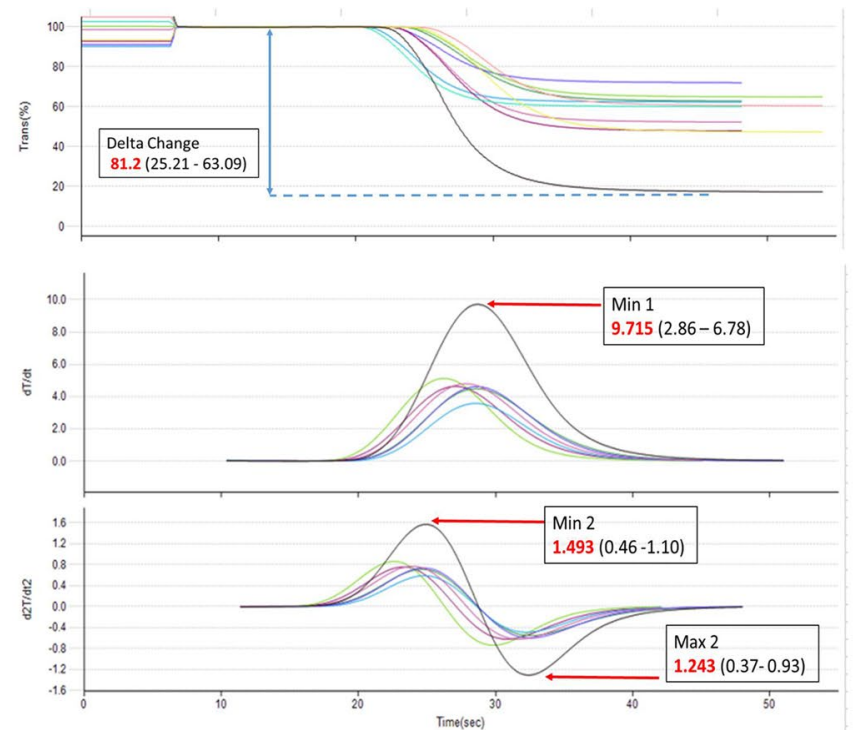

A
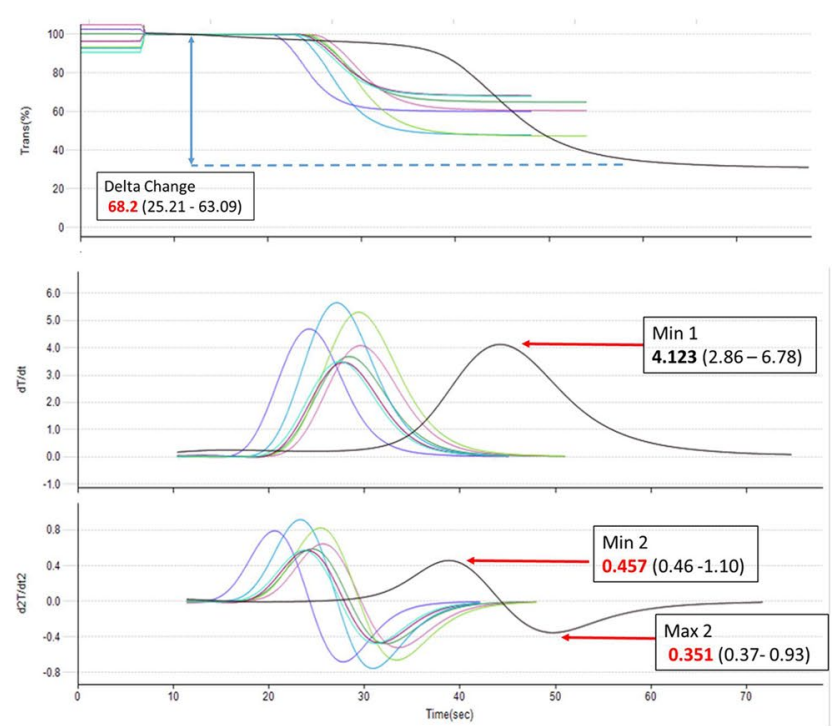

B

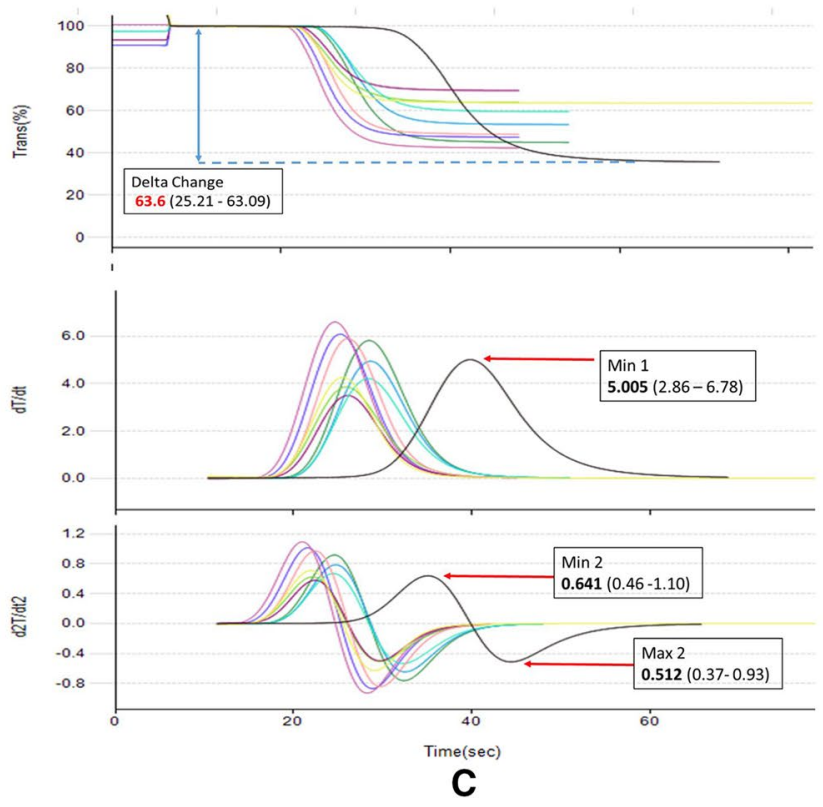

thromboprophylaxis and the other sustained a fatal intracranial haemorrhage on Day 4 of ECMO while on therapeutic unfractionated heparin. Major bleeding in the critically ill with COVID-19, though rare, is significant clinically given the high morbidity and mortality associated with it. A bleeding diathesis could have resulted from coexisting sepsis induced coagulopathy or pulmonary intravascular coagulopathy; precipitated by refractory hypoxaemia, or alternatively caused by local factors such as endothelial damage. Yang et al. [26] described a $4 \%$ incidence (2 out of 25 patients) of gastrointestinal bleeding in critically ill patients while Xiao et al. [27] reported a $13.7 \%$ incidence (10 out of 73 patients) but did not discuss severity. Xiao et al. showed epithelial damage by the SARS-CoV-2 virus as an additional factor. They demonstrated fecal-oral transmission of the SARS-CoV-2 virus as a route of transmission, by means of entry into gastrointestinal epithelial cells by the angiotensin converting enzyme 2 (ACE2) protein. Mucosal damage to the esophagus was demonstrated with SARS-CoV-2 positive staining in tissues obtained from the esophagus, stomach, duodenum, and rectum. This may possibly explain why in critically ill patients with COVID-19 infection, gastrointestinal mucosal damage from SARS-CoV-2 infection combined with stress or uremic gastritis, cell necrosis from tissue hypoxia may cause 
४Fig. 1 a Hypercoagulable aPTT Clot Waveform tracing and parameters of a 35-year-old male with severe COVID-19 infection complicated by acute right ischaemic limb secondary to extensive thromboembolic disease in the abdominal aorta, right external iliac and right popliteal arteries. *Patient's clot waveform tracing in black, healthy controls in multicolour (for reference). Patient's haemostatic and clot waveform parameters: PT $14.2 \mathrm{~s}(11.7-14.0 \mathrm{~s})$, aPTT $38.7 \mathrm{~s}$ (27.0-37.0 s), fibrinogen $5.0 \mathrm{~g} / \mathrm{L}(1.8-4.5 \mathrm{~g} / \mathrm{L})$, D-Dimer $2.26 \mu \mathrm{g} /$ $\mathrm{ml}(<0.50 \mu \mathrm{g} / \mathrm{ml})$, Platelets $337 \times 10^{9} / \mathrm{L}\left(150-360 \times 10^{9} / \mathrm{L}\right)$. Factor II $122 \%$ (70-120\%), Factor VIII 196\% (60-150\%), Factor $\times 106 \%(70-$ $120 \%)$, vWF $200 \%$ (56-160\%), anti-thrombin III 74\% (80-130\%), Protein C 61\% (70-150\%), Protein S 79\% (55-130\%). Absent lupus anticoagulant and antiphospholipid antibodies. CWA: Min1 9.715\%/s $(2.86-6.78 \% / \mathrm{s}), \operatorname{Min} 21.493 \% / \mathrm{s}^{2}\left(0.46-1.10 \% / \mathrm{s}^{2}\right), \operatorname{Max} 21.243 \% / \mathrm{s}^{2}$ $\left(0.37-0.93 \% / \mathrm{s}^{2}\right)$, Delta change $81.2 \%(25.21-63.09 \%)$. b aPTT Clot Waveform tracing of a 40 -year-old male on mechanical ventilation for severe COVID-19 pneumonia, Day 18 of illness (Day 2 ECMO), with probable pulmonary intravascular coagulopathy with concurrent bacterial pneumonia and sepsis induced coagulopathy, not initiated on anticoagulation yet. Clots were seen in the ECMO oxygenator as well as the dialysis circuit. *Patient's clot waveform tracing in black, healthy controls in multicolour (for reference). Patient's haemostatic and clot waveform parameters. PT $18.2 \mathrm{~s}(11.7-14.0 \mathrm{~s})$, aPTT $53.2 \mathrm{~s}$ (27.0-37.0 s), fibrinogen $3.8 \mathrm{~g} / \mathrm{L}(1.8-4.5 \mathrm{~g} / \mathrm{L})$, D-Dimer $>20 \mu \mathrm{g} / \mathrm{ml}$ $(<0.5 \mu \mathrm{g} / \mathrm{ml})$, Platelets $115 \times 10^{9} / \mathrm{L}\left(150-360 \times 10^{9}\right)$. Factor II $46 \%$ (70-120\%), Factor V 68\% (70-120\%), Factor VIII 196\% (60-150\%), Factor IX 115\% (60-150\%) Factor $\times 68 \%(70-120 \%)$, vWF $387 \%$ (56-160\%), anti-thrombin III 54\% (80-130\%), Protein C 28\% (70$130 \%$ ), Protein S 19\% (55-130\%). Lupus anticoagulant moderately present and anti cardiolipin $\operatorname{IgG}<20$ ( $0-20$ GPL units), anti cardiolipin IgM 23 (0-20 MPL units), anti B2 glycoprotein-1 $<2$ RU/ml (0-20 RU/ml). CWA: Min1 4.123\%/s (2.86-6.78\%/s), Min2 0.457\%/ $\mathrm{s}^{2}\left(0.46-1.10 \% / \mathrm{s}^{2}\right), \operatorname{Max} 20.351 \% / \mathrm{s}^{2}(0.37-0.93)$, Delta change $68.2 \%$ (25.21-63.09\%). c aPTT Clot Waveform tracing of a 73-year-old male on Day 13 of illness with severe COVID-19 pneumonia with probable pulmonary intravascular coagulopathy and concurrent sepsis induced coagulopathy from stenotrophomonas maltophilia bacteraemia and ventilator associated pneumonia. *Patient's clot waveform tracing in black, healthy controls in multicolour (for reference). Patient's haemostatic and clot waveform parameters. PT $15.3 \mathrm{~s}$ (11.7$14.0 \mathrm{~s})$, aPTT $41.3 \mathrm{~s}(27.0-37.0 \mathrm{~s})$, fibrinogen $4.0 \mathrm{~g} / \mathrm{L}(1.8-4.5 \mathrm{~g} / \mathrm{L})$, D-Dimer $>20 \mu \mathrm{g} / \mathrm{ml}(<0.5 \mu \mathrm{g} / \mathrm{ml})$, Platelets $120 \times 10^{9} / \mathrm{L}$. Factor II $56 \%$ (70-120\%), Factor V $93 \%$ (70-120\%), Factor VIII 195\% (60$150 \%)$, Factor IX $105 \%$ (60-150\%), Factor $\times 68 \%$ (70-120\%), Factor XI 47\% (60-150\%), vWF 200\% (56-160\%), anti-thrombin III 74\% (80-130\%), Protein C 61\% (70-150\%), Protein S 79\% (55-130\%). Lupus anticoagulant and antiphospholipid antibodies absent. CWA: Min1 5.005\%/s (2.86-6.78\%/s), Min2 $0.641 \% / \mathrm{s}^{2} \quad(0.46-1.10 \% /$ $\left.\mathrm{s}^{2}\right)$, Max $20.512 \% / \mathrm{s}^{2}\left(0.37-0.93 \% / \mathrm{s}^{2}\right)$, Delta change $63.6 \%(25.21-$ $63.09 \%)$

gastrointestinal mucosal cellular injury, resulting in major gastrointestinal tract bleeding.

While ICH is a known complication of patients on ECMO, our patient who had catastrophic ICH had close monitoring of anti Xa levels which were within the therapeutic range (aPTT results were not used for titration of unfractionated heparin given the presence of a lupus anticoagulant) with mild thrombocytopenia while on therapeutic anticoagulation. Early reports of brain imaging findings in COVID19 patients showed ischaemic and haemorrhagic features $[28,29]$, and more recently acute haemorrhagic necrotizing encephalopathy [30], demyelinating lesions, diffuse leukoencephalopathy and microhaemorrhages [31]. These diffuse leukoencephalopathies and microhaemorrhages have been postulated to be late hypoxia related complications of critically ill patients with COVID-19 who were not in DIC, and we postulate could possibly result in catastrophic ICH while on anticoagulation.

Lupus anticoagulant was detected in 6 out of $12(50 \%)$ of our patients, with lower number of patients who were positive for anti-cardiolipin and anti- $\beta 2$ glycoprotein antibodies ( 2 out of 4 patients who were tested). The increased incidence of a lupus anticoagulant in critically ill patients with COVID-19 was also seen in ICU studies by other centres $[20,32]$ with a higher prevalence of $87.7-88 \%$. The presence of anti-phospholipid antibodies is likely caused by an immune dysregulation and cytokine storm. Given the prolongation of aPTT due to the lupus anticoagulant, monitoring with anti-Xa levels is recommended while patients are on therapeutic anticoagulation although the significance of these antibodies predisposing to development of thrombosis in COVID-19 is unclear and requires further evaluation.

The main limitations of our study are firstly, the small patient number which results in the inability to generalize findings. Secondly, as this study is from an ICU cohort, the threshold for ICU admission may also be a confounder as countries with a lower threshold for early ICU admission (ie. Singapore) for patients early in their illness may see a higher proportion of patients who have a hypercoagulable state whereas countries who admit patients later in their illness (i.e United States, United Kingdom, Italy, Brazil) may see patients admitted to ICU with severe COVID-19 complicated with SIC or PIC, which may result in higher bleeding manifestations [33,34] due to consumptive coagulopathy.

Lastly, the variable time of assessment since onset of illness ranging between 9 and 20 days is a significant confounder, as the hypercoagulable state in COVID-19 is observed early during SARS-CoV-2 infection, while in patients who are not on thromboprophylaxis or already on long term anticoagulation, this may then progress on to pulmonary intravascular coagulopathy (PIC), a consumptive coagulopathy, with excessive activation of the coagulation system resulting in consumption of both clotting factors and platelets, shifting the hypercoagulable state into a hypocoagulable state [35]. Hence the different timing of assessment may result in discordant findings in both haemostatic parameters and clot waveform analysis. Our 12 patients were not on thromboprophylaxis at time of assessment, and those who had their haemostatic parameters and CWA assessed in the later part of their hospitalization could have developed PIC. Pathologic evidence from autopsy series demonstrating macroscopic findings of lung edema and patchy haemorrhages, microscopic findings of pulmonary endotheliitis [36], extensive fibrin thrombi in distended small vessels and capillaries [37, 38], increased numbers of megakaryocytes in 
Table 3 Comparison of aPTT Clot Waveform Parameters between ICU Patients and Healthy Controls

\begin{tabular}{|c|c|c|c|c|c|c|c|}
\hline \multirow[t]{2}{*}{ Clot Waveform Parameters } & \multicolumn{3}{|c|}{ Patients $(\mathrm{N}=12)$} & \multicolumn{3}{|c|}{ Controls $(\mathrm{N}=124)$} & \multirow[t]{2}{*}{ P-value } \\
\hline & No. $(\%)$ & Median (IQR) & Mean (SD) & No. $(\%)$ & Median (IQR) & Mean (SD) & \\
\hline $\operatorname{Min} 1, \% / \mathrm{s}$ & & $9.3(7.1,9.9)$ & $8.3(2.1)$ & & $4.7(4.1,5.4)$ & $4.8(0.9)$ & $<0.001$ \\
\hline $\operatorname{Min} 2, \% / s^{2}$ & & $1.5(1.0,1.6)$ & $1.3(0.4)$ & & $0.8(0.6,0.9)$ & $0.8(0.2)$ & $<0.001$ \\
\hline $\operatorname{Max} 2, \% / s^{2}$ & & $1.3(0.8,1,4)$ & $1.1(0.4)$ & & $0.6(0.5,0.7)$ & $0.6(0.1)$ & 0.0001 \\
\hline Delta change, $\%$ & & $87.8(70.2,91.8)$ & $82.8(11.5)$ & & $43.0(37.8,49.5)$ & $44.2(9.5)$ & $<0.001$ \\
\hline \multicolumn{8}{|l|}{ CWA > ULRR } \\
\hline $\operatorname{Min} 1>6.78$ & $10(83.3)$ & & & $5(4.0)$ & & & $<0.001$ \\
\hline Min $2>1.10$ & $9(75.0)$ & & & $3(2.4)$ & & & $<0.001$ \\
\hline $\operatorname{Max} 2>0.93$ & $8(66.7)$ & & & $3(2.4)$ & & & $<0.001$ \\
\hline Delta change $>63.09$ & $12(100.0)$ & & & $7(5.7)$ & & & $<0.001$ \\
\hline
\end{tabular}

ULRR: Upper limit of reference interval. $\mathrm{P}<0.05$ was considered statistically significant

Table 4 Comparison of PT Clot Waveform Parameters between ICU Patients and Healthy Controls

\begin{tabular}{|c|c|c|c|c|c|c|c|}
\hline \multirow[t]{2}{*}{ Clot Waveform Parameters } & \multicolumn{3}{|c|}{ Patients $(\mathrm{N}=12)$} & \multicolumn{3}{|c|}{ Controls $(\mathrm{N}=124)$} & \multirow[t]{2}{*}{ P-value } \\
\hline & No. $(\%)$ & Median (IQR) & Mean (SD) & No. $(\%)$ & Median (IQR) & Mean (SD) & \\
\hline $\operatorname{Min} 1, \% / \mathrm{s}$ & & $10.3(7.1,11.1)$ & $9.2(2.8)$ & & $3.6(3.2,4.1)$ & $3.8(0.9)$ & $<0.001$ \\
\hline $\operatorname{Min} 2, \% / s^{2}$ & & $5.2(3.6,5.7)$ & $4.6(1.5)$ & & $1.9(1.6,2.1)$ & $1.9(0.5)$ & $<0.001$ \\
\hline $\operatorname{Max} 2, \% / s^{2}$ & & $3.8(2.6,4.2)$ & $3.4(1.1)$ & & $1.5(1.3,1.7)$ & $1.5(0.4)$ & $<0.001$ \\
\hline Delta change, $\%$ & & $33.0(23.9,37.2)$ & $30.7(8.9)$ & & $11.4(10.1,12.8)$ & $11.9(2.7)$ & $<0.001$ \\
\hline \multicolumn{8}{|l|}{ CWA > ULRR } \\
\hline Min $1>5.67$ & $10(83.3)$ & & & $7(5.7)$ & & & $<0.001$ \\
\hline $\operatorname{Min} 2>2.93$ & $10(83.3)$ & & & $7(5.7)$ & & & $<0.001$ \\
\hline $\operatorname{Max} 2>2.35$ & $10(83.3)$ & & & $7(5.7)$ & & & $<0.001$ \\
\hline Delta change $>17.28$ & $11(91.7)$ & & & $7(5.7)$ & & & $<0.001$ \\
\hline
\end{tabular}

ULRR: Upper limit of reference interval. $\mathrm{P}<0.05$ was considered statistically significant. Reference intervals for Clot Waveform parameters were established locally based on 124 healthy controls in accordance with the Clinical and Laboratory Standards Institute guidelines

the pulmonary tissue support the lungs as the immunothrombotic epicentre in COVID-19. The result cumulates in end organ complications of thrombosis as seen in cases of lower limb ischaemia and deep venous thrombosis, acute myocardial infarctions and cerebrovascular accidents. Therefore, the treatment strategy on presentation of COVID-19 could be thromboprophylaxis with the blockade of hypercoagulation and thrombin generation with low molecular weight heparin or unfractionated heparin $[39,40]$. Further prospective randomized control trials are awaited to determine if intermediate or full dose anticoagulation strategy will provide a decreased incidence of thrombotic events and survival benefit in critically ill patients.

\section{Conclusions}

In summary, the use of haemostatic parameters and CWA further substantiate the evidence for a hypercoagulability state in severe COVID-19. Our analysis showed that elevated
CWA parameters together with elevated D-dimer, Factor VIII, vWF antigen and fibrinogen levels, support the presence of COVID-19-associated coagulopathy. CWA seems to reflect a hypercoagulable state in critically ill patients with COVID-19 as shown by elevated clot waveform parameters (min1, min2, max2 and delta change) with good correlation with elevated factor VIII, vWF antigen, D-dimer and fibrinogen levels. CWA parameters may hence offer a novel approach for assessment of COVID-19-associated hypercoagulabilty apart from viscoelastic tests. It is easily performed on automated analyzers with optical detection systems using standard PT and aPTT reagents and is not associated with additional costs. Patients with concurrent PIC or SIC with COVID-19-associated coagulopathy are more likely to show less elevated or low-normal CWA parameters. Major bleeding associated with a consumptive coagulopathy from SIC, PIC or local factors from endothelial damage from SARS$\mathrm{CoV}$ virus infection can occur in critically ill patients with severe COVID-19, causing significant morbidity and mortality. Further randomized studies which include both ICU 
and non ICU patients and risk prediction models for venous thromboembolism and arterial thrombosis are needed to establish the utility of standard haemostatic tests and global haemostatic tests such as viscoelastic tests, CWA and thrombin generation in patients with COVID-19 infection that may help to establish appropriate thromboprophylactic regimes, and to manage prothrombotic and bleeding risks.

Acknowledgements The COVID-19 Clotting and Bleeding Team at the National Centre for Infectious Diseases, Singapore greatly appreciates the efforts of our fellow healthcare workers and the support of their families during this pandemic. Special thanks to Sysmex Corporation (Japan) for their technical support and loan of CN-6000.

Author contribution BE Fan, $\mathrm{J} \mathrm{Ng}$ and YW Chia conceived the study. BE Fan collated the data and wrote the manuscript. CLL Sum, HT Tan and MK Ang performed the coagulation tests. GH Lim performed the statistical analysis. All authors contributed substantially in analysing the data and editing the manuscript.

\section{Compliance with ethical standards}

Conflict of interest The authors declare that they have no conflict of interest. No funding for this study was obtained.

Ethical approval This study was approved by the National Healthcare Group Domain Specific Review Board (DSRB).

\section{References}

1. Iba T, Levy JH, Levi M, Connors JM, Thachil J (2020) Coagulopathy of coronavirus disease 2019 [published online ahead of print, 2020 May 27]. Crit Care Med. https://doi.org/10.1097/ CCM.0000000000004458

2. Becker RC (2020) COVID-19 update: COVID-19-associated coagulopathy. J Thromb Thrombolysis 50(1):54-67. https://doi. org/10.1007/s11239-020-02134-3

3. Thachil J, Tang N, Gando S et al (2020) ISTH interim guidance on recognition and management of coagulopathy in COVID-19. J Thromb Haemost 18(5):1023-1026. https://doi.org/10.1111/ jth. 14810

4. Hunt B, Retter A, McClintock C (2020) Practical guidance for the prevention of thrombosis and management of coagulopathy and disseminated intravascular coagulation of patients infected with COVID-19. https://b-s-h.org.uk/media/18171/th-and-covid -25-march-2020-final.pdf

5. Kreuziger LB, Lee A, Garcia D, et al (2020) COVID-19 and VTE/ anticoagulation: frequently asked questions (Version 4.0; last reviewed July 20, 2020) https://www.hematology.org/covid-19/ covid-19-and-vte-anticoagulation

6. Becker RC (2020) COVID-19-associated vasculitis and vasculopathy. J Thromb Thrombolysis. https://doi.org/10.1007/s1123 9-020-02230-4

7. Fogarty H, Townsend L, Ni Cheallaigh C et al (2020) COVID19 coagulopathy in Caucasian patients. Br J Haematol 189(6):10441049. https://doi.org/10.1111/bjh.16749

8. Bikdeli B, Madhavan MV, Jimenez D et al (2020) COVID19 and thrombotic or thromboembolic disease: implications for prevention, antithrombotic therapy, and follow-up: JACC
State-of-the-art review. J Am Coll Cardiol 75(23):2950-2973. https://doi.org/10.1016/j.jacc.2020.04.031

9. Panigada M, Bottino N, Tagliabue P et al (2020) Hypercoagulability of COVID-19 patients in intensive care unit: a report of thromboelastography findings and other parameters of hemostasis. J Thromb Haemost 18(7):1738-1742. https://doi.org/10.1111/ jth. 14850

10. Pavoni V, Gianesello L, Pazzi M, Stera C, Meconi T, Frigieri FC (2020) Evaluation of coagulation function by rotation thromboelastometry in critically ill patients with severe COVID-19 pneumonia. J Thromb Thrombolysis 50(2):281-286. https://doi. org/10.1007/s11239-020-02130-7

11. Tan CW, Low JGH, Wong WH, Chua YY, Goh SL, Ng HJ (2020) Critically ill COVID-19 infected patients exhibit increased clot waveform analysis parameters consistent with hypercoagulability. Am J Hematol 95(7):E156-E158. https://doi.org/10.1002/ ajh. 25822

12. Lancé MD (2015) A general review of major global coagulation assays: thrombelastography, thrombin generation test and clot waveform analysis. Thromb J 13:1. Published 2015 Jan 12. https ://doi.org/10.1186/1477-9560-13-1

13. Sevenet PO, Depasse F (2017) Clot waveform analysis: where do we stand in 2017? Int J Lab Hematol 39(6):561-568. https://doi. org/10.1111/ijlh.12724

14. Lee LH, Gallus A, Jindal R, Wang C, Wu CC (2017) Incidence of venous thromboembolism in Asian populations: a systematic review. Thromb Haemost 117(12):2243-2260. https://doi. org/10.1160/TH17-02-0134

15. Shima M, Thachil J, Nair SC (2013) Srivastava a; scientific and standardization committee. Towards standardization of clot waveform analysis and recommendations for its clinical applications. J Thromb Haemost 11(7):1417-1420. https://doi.org/10.1111/ jth.12287

16. Fan BE, Chia YW, Sum CLL et al (2020) Global haemostatic tests in rapid diagnosis and management of COVID-19 associated coagulopathy in acute limb ischaemia [published online ahead of print, 2020 May 30]. J Thromb Thrombolysis:1-6. https://doi. org/10.1007/s11239-020-02165-w

17. McGonagle D, O’Donnell JS, Sharif K, Emery P, Bridgewood C (2020) Why the immune mechanisms of pulmonary intravascular coagulopathy in COVID-19 pneumonia are distinct from macrophage activation syndrome with disseminated intravascular coagulation. Autoimmun Rev. https://doi.org/10.1016/j.autre v.2020.102537

18. Zakariah AN, Cozzi SM, Van Nuffelen M, Clausi CM, Pradier O, Vincent JL (2008) Combination of biphasic transmittance waveform with blood procalcitonin levels for diagnosis of sepsis in acutely ill patients. Crit Care Med 36(5):1507-1512. https://doi. org/10.1097/CCM.0b013e3181709f19

19. Ten Cate $\mathrm{H}$ (2004) The biphasic waveform in plasma: identifying the sepsis-coagulation crossroad? J Thromb Haemost 2(9):15331534. https://doi.org/10.1111/j.1538-7836.2004.00878.x

20. Harzallah I, Debliquis A, Drénou B (2020) Lupus anticoagulant is frequent in patients with COVID-19. J Thromb Haemost 18(8):2064-2065. https://doi.org/10.1111/jth.14867

21. Helms J, Tacquard C, Severac F et al (2020) High risk of thrombosis in patients with severe SARS-CoV-2 infection: a multicenter prospective cohort study. Intensive Care Med 46(6):1089-1098. https://doi.org/10.1007/s00134-020-06062-x

22. Tan CW, Cheen MHH, Wong WH et al (2019) Elevated activated partial thromboplastin time-based clot waveform analysis markers have strong positive association with acute venous thromboembolism. Biochem Med (Zagreb) 29(2):020710. https://doi. org/10.11613/BM.2019.020710

23. Su Z, Braun PJ, Klemp KF, Baker KR, Thames EH, Ortel TL (2002) Abnormal optical waveform profiles in coagulation assays 
from patients with antiphospholipid antibodies. Blood Coagul Fibrinolysis 13(1):7-17. https://doi.org/10.1097/00001721-20020 1000-00002

24. Ogiwara K, Nogami K, Nishiya K, Shima M (2010) Plasmininduced procoagulant effects in the blood coagulation: a crucial role of coagulation factors V and VIII. Blood Coagul Fibrinolysis 21(6):568-576. https://doi.org/10.1097/MBC.0b013e32833c9a9f

25. Tang N, Li D, Wang X, Sun Z (2020) Abnormal coagulation parameters are associated with poor prognosis in patients with novel coronavirus pneumonia. J Thromb Haemost 18(4):844-847. https://doi.org/10.1111/jth.14768

26. Yang XB, Yu Y, Xu JQ, Shu HQ, Xia JA, Liu H et al (2020) Clinical course and outcomes of critically ill patients with SARSCoV-2 pneumonia in Wuhan, China: a single-centered, retrospective, observational study. Lancet Respir Med 8:P475-P481

27. Xiao F, Tang M, Zheng X, Liu Y, Li X, Shan H (2020) Evidence for gastrointestinal infection of SARS-CoV-2. Gastroenterology 158(6):1831-1833.e3. https://doi.org/10.1053/j.gastr o.2020.02.055

28. Radmanesh A, Raz E, Zan E, Derman A, Kaminetzky M (2020) Brain imaging use and findings in COVID-19: a single academic center experience in the epicenter of disease in the United States. AJNR Am J Neuroradiol 41(7):1179-1183. https://doi. org/10.3174/ajnr.A661

29. Mao L, Jin H, Wang $M$ et al (2020) Neurologic manifestations of hospitalized patients with coronavirus disease 2019 in Wuhan, China [published online ahead of print, 2020 April 10]. JAMA Neurol 77(6):1-9. https://doi.org/10.1001/jamaneurol.2020.1127

30. Poyiadji N, Shahin G, Noujaim D, Stone M, Patel S, Griffith B (2020, March) COVID-19-associated acute hemorrhagic necrotizing encephalopathy: CT and MRI features. Radiology 31:201187. https://doi.org/10.1148/radiol.2020201187

31. Radmanesh A, Derman A, Lui YW et al (2020, May) COVID19-associated diffuse leukoencephalopathy and microhemorrhages. Radiology 2020:202040. https://doi.org/10.1148/radio 1.2020202040

32. Siguret V, Voicu S, Neuwirth M et al (2020) Are antiphospholipid antibodies associated with thrombotic complications in critically ill COVID-19 patients? [published online ahead of print, 2020
July 8]. Thromb Res 195:74-76. https://doi.org/10.1016/j.throm res.2020.07.016

33. Lucatelli P, De Rubeis G, Citone M et al (2020) Heparin-related major bleeding in COVID-19-positive patient: perspective from the outbreak. Cardiovasc Intervent Radiol 43(8):1216-1217. https ://doi.org/10.1007/s00270-020-02532-3

34. Conti CB, Henchi S, Coppeta GP, Testa S, Grassia R (2020) Bleeding in COVID-19 severe pneumonia: the other side of abnormal coagulation pattern? Eur J Intern Med 77:147-149. https:// doi.org/10.1016/j.ejim.2020.05.002

35. Levi M (2008) The coagulant response in sepsis. Clin Chest Med 29(4):627-viii. https://doi.org/10.1016/j.ccm.2008.06.006

36. Varga Z, Flammer AJ, Steiger P et al (2020) Endothelial cell infection and endotheliitis in COVID-19. Lancet 395(10234):14171418. https://doi.org/10.1016/S0140-6736(20)30937-5

37. Dolhnikoff M, Duarte-Neto AN, de Almeida Monteiro RA et al (2020) Pathological evidence of pulmonary thrombotic phenomena in severe COVID-19. J Thromb Haemost 18(6):1517-1519. https://doi.org/10.1111/jth.14844

38. Ackermann M, Verleden SE, Kuehnel M et al (2020) Pulmonary vascular endothelialitis, thrombosis, and angiogenesis in COVID19. N Engl J Med 383(2):120-128. https://doi.org/10.1056/ NEJMoa2015432

39. Marchandot B, Trimaille A, Curtiaud A, Matsushita K, Jesel L, Morel O (2020) Thromboprophylaxis: balancing evidence and experience during the COVID-19 pandemic [published online ahead of print, 2020 July 21]. J Thromb Thrombolysis:1-10. https ://doi.org/10.1007/s11239-020-02231-3

40. Thachil J, Juffermans NP, Ranucci M, Connors JM, Warkentin TE, Ortel TL, Levi M, Iba T, Levy JH (2020) ISTH DIC subcommittee communication on anticoagulation in COVID-19. J Thromb Haemost. Accepted Author Manuscript. https://doi.org/10.1111/ jth.15004

Publisher's Note Springer Nature remains neutral with regard to jurisdictional claims in published maps and institutional affiliations. 